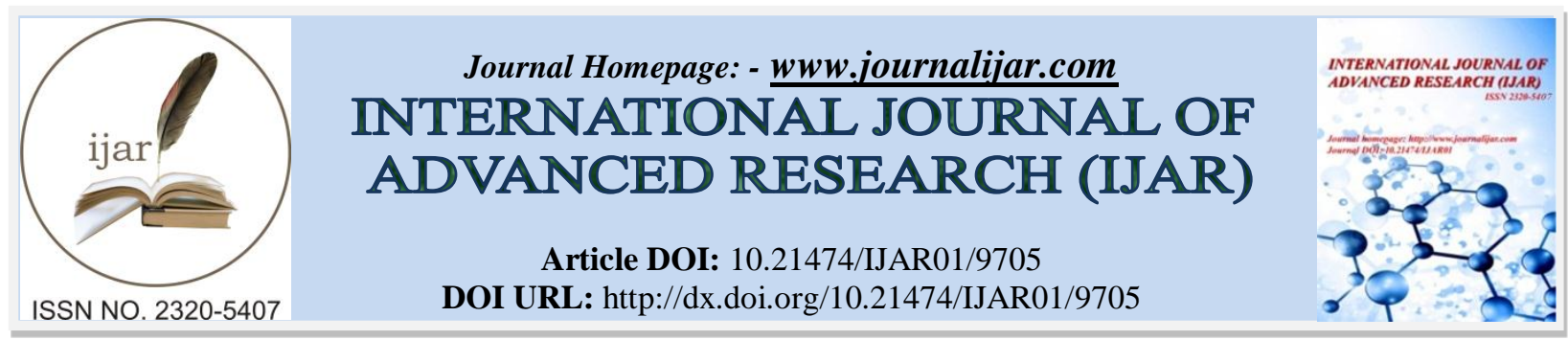

RESEARCH ARTICLE

\title{
THE PREVALENCE AND ASSOCIATED FACTORS OF SCREEN TIME AMONG INTERMEDIATE SCHOOL STUDENTS IN MAKKAH AL-MOKARRAMAH, SAUDI ARABIA, 2019.A CROSS- SECTIONAL STUDY.
}

Ebtihal Ahmad Alwadie.

Family medicine resident in Security forces Hospital Family medicine Program in Al-Dammam, Saudi Arabia.

\section{Manuscript Info}

Manuscript History

Received: 08 July 2019

Final Accepted: 10 August 2019

Published: September 2019

Key words:-

Screen time, electronic devices, adolescents.

\section{Abstract}

Aim of study: To assess screen-time among intermediate school students in Makkah Al-Mokarramah.

Methodology: Following a cross-sectional study design in in Alawali District, Makkah City, a total of 175 intermediate school students (85 boys and 90 girls) were included in the present study. A selfadministered questionnaire was used.

Results: About three-fourths of students (71.4\%) use electronic devices. The average duration of daily use of electronic devices was $\leq$ 1 hour among $42.9 \%$ of students, $2-4$ hours among $25.7 \%$ of students, while it was 4-6 hours among $1.7 \%$ and $>7$ hours among $1.1 \%$. The smartphones were the most frequently used electronic devices $(69.7 \%)$, followed by the television $(52.6 \%)$, videogames $(52 \%)$, tablets $(40 \%)$, laptops/computers (32\%) and the cellular phones (10.9\%). Use of electronic devices increases significantly at weekends $(\mathrm{p}<0.001)$. Prolonged (>8 hours) physical activity was significantly more during weekends $(9.1 \%$ vs. $0 \%, \mathrm{p}<0.001)$. On the other hand, duration of sleep did not differ during weekdays and weekends. Use of electronic devices was significantly higher among male students than female students (78.8\% and $64.4 \%$, respectively, $\mathrm{p}=0.035)$. Students whose GPA was $>95 \%$ had significantly highest use of electronic devices $(85.3 \%$, $\mathrm{p}=0.024)$. Use of electronic devices did not differ significantly according to care takers' personal characteristics or family characteristics.

Conclusions: One-fourth intermediate school students in Makkah AlMokarramah, have prolonged daily screen time. The most frequently used electronic devices are mainly smartphones, TV, videogames, tablets, and laptops or computers. Use of electronic devices increases during their weekends and entertainment is the most frequent purpose for using electronic devices for more than 8 hours. Screen time is significantly higher among boys and those with higher GPA.

Recommendations: Immediate and comprehensive actions are needed to diminish adolescents' screen time. TV-watching time can be minimized through school-based educational components assisted by parental involvement with students' homework. Health education of adolescents and their parents to raise their awareness regarding health risks associated with high screen time and how they can positively 
influence their children by enforcing family rules to limit using electronic devices.

Copy Right, IJAR, 2019,. All rights reserved.

\section{Introduction:-}

"Screen time" is a term referred to time spent in front of a screen, such as watching TV, working on a computer, or video games playing. It is a sedentary behavior, in other words being physically inactive while sitting down with minimum energy consumed during screen time.(1)

In the past 50 years, thousands of research studies have revealed that the media can be a powerful teacher of children and adolescents, at the same time have a profound impact on their health. Sedentary activity is a major risk factor for chronic diseases, Children and adolescents per se need enough sleep hours, exercise, and proper nutrition for healthy growth. Excessive 'screen time' interferes with these essential needs. Studies show that excess 'screen time' is linked to obesity, declining levels of fitness and nutrition, and sleeping problems. (2)

The American Academy of Family Physician and the American Academy of Pediatrics (AAP) recommend no more than two h/day for children 2 years and older(10). On Oct. 2016, American Academy of Pediatrics renew their recommendations of children and screen time, some of the recommendations regarding adolescent were:

1. For children six years and older, place consistent limits on the time spent in front of screens and make sure media does not take the place of sleeping time and the essential healthy lifestyle.

2. Designate screen-free times together, such as the family meal, as well as screen-free locations at home. With continuous emphasizing about online citizenship and safety, including treating others with respect all the time. (3)

3. A study conducted in the United States about Screen Time in US Children, showed that $49 \%$ of US 6 to 17 years old exceeded AAP screen time guidelines, and Screen time was inversely related to the frequency of physical activity. (4)

4. An article in the United Kingdom, considered screen time among youth concluded that: Excluding viewing time outside the home, such as mobile-device screen activities, the average screen time in the home for British adolescents is approximately six hours a day, and this is rising significantly. Canadian children spend more than seven h/day. (5)

5. One study reported that each additional hour of TV viewing per week increased the risk of obesity by $2 \%$ (6), another study showed that excessive TV viewing in adolescence is a risk factor for the development of depression in young adulthood(7).

In Saudi Arabia, a study had done about Smartphone addiction among university students in Riyadh, indicated that $82.1 \%$ of the study subjects had been using a smartphone for more than three years.

Approximately $29 \%$ of the survey subjects and $15.3 \%$ agree and strongly agreed respectively, that their sleep time had decreased since they began using their smartphones. 34\% agreed that they lacked energy the next day; Increase fast food eating more than before using their smartphones; therefore they had gained weight; $16 \%$ and $7.3 \%$ adversely affected academic achievement, and less exercise compared with before their smartphone use(8).

Another survey conducted among children of school-age in Saudi Arabia studied TV watching and obesity confirms that a substantial percentage of school-age children watching television for more than three hours on weekdays and at weekends, represents an important risk factor for obesity in children of school-age (9).

Another study about the behavioral problems associated with using smart phones among children from the parents' point of view, showed that most behavioral problems resulting from using smart phones were the social, educational and psychological problems. In addition, showed significant statistical differences in behavioral problems resulting from using smart phones due to gender in favor of males. Moreover, there were significant statistical differences due to age in favor of ages (eight - twelve years old), and the hours of use in favor of (1-3 hours) and (> 3 hours). (10) 
A study about Physical activity, sedentary behaviors and dietary habits among Saudi adolescents relative to age, gender and region, showed that a very high proportion ( $84 \%$ for males and $91.2 \%$ for females) spent $>2 \mathrm{hs}$ on screen time daily.(11)

\section{Study Rationale}

The whole world, with its good and bad, are available now between our children hands. Additionally, screen-time is a sedentary lifestyle, which is a major risk factor for chronic disease. Children and adolescents especially need enough nutrition, exercise, and sleep for healthy life, excess screen time interferes with sports, community services and family time.

To the best of the researcher's knowledge, no previous study has been conducted to assess TV viewing, videogames playing, and computer use in children and adolescents in Saudi Arabia. Moreover, the researcher has a particular interest in this issue.

\section{Aim of the study}

To assess screen-time among intermediate school students in Makkah Al-Mokarramah.

\section{Objectives:-}

1. To assess the screen-time among intermediate school students in Makkah Al-Mokarramah.

2. To determine the factors associated with screen time, among intermediate school students in Makkah AlMokarramah.

\section{Methodology:-}

Study Design

Analytical cross-sectional study.

\section{Study Area}

Makkah City is the center of the Islamic world and the birthplace of both the Prophet Muhammad and the religion he founded. It is located in the western area in the Kingdom of Saudi Arabia. It is located $70 \mathrm{~km}$ from Jeddah City, at an altitude of $277 \mathrm{~m}$ above sea level. In 2012, its resident population was 2 million, every year during the Hajj; Makkah Al-Mokarramah receives millions of Muslims.

Regarding education, the first school founded in Makkah Almukarramah was Madrasat al-Falāh in 1911. Nowadays, the educational system in Makkah Al-Mokarramah has many public and private schools for both males and females. In 2005, there were 532 public and private schools for males and 681 schools for female students. For higher education, there is only Umm Al-Qura University, established in 1949 and became a public university in 1979.

This study will be conducted in all Alawali intermediate schools. There are five public and private intermediate schools in Alawali Distract, two for male students and three for female students.

\section{Study Population}

All students attending intermediate schools in Alawali District in Makkah Al-Mokarramah City at the time of the survey.

\section{Inclusion criteria}

All intermediate school students, Saudi and non-Saudi, who study in the Alawali District in Makkah Al-Mokarrmah City at the time of the survey were included.

\section{Exclusion criteria}

1. Caregivers or students who refused to participate or do not give the consent.

2. Students who have a disability or psychological problem.

3. Students who were absent during the period of study. 


\title{
Sample size
}

The minimum sample size for the present study was calculated by using "Raosoft" sample size calculator (12), and adding $10 \%$, according to the following:

1. Study population: The total number of students in all intermediate schools in Alawali are 1500 students.

2. Prevalence of the study problem: Physical activity, sedentary behaviors and dietary habits among Saudi adolescents relative to age, gender and region, showed that a very high proportion ( $84 \%$ for males and $91.2 \%$ for females) spent more than 2 hours' screen-time daily.(10)

3. Confidence level: $95 \%$

4. Error: $5 \%$

5. Accordingly, the minimum sample size was calculated to be 126 students.

\section{Sampling technique}

The researcher followed a simple random sampling technique. Using lists of students' names, each student was given a unique serial number. Then, the study sample was randomly decided using the True Random Number generator at "Random.org". (13)

\section{Data collection tool (instrument)}

A self-administered questionnaire was used. The questionnaire was taken from another similar study, and necessary modifications were made by the researcher to meet the study objectives. The study questionnaire was translated into Arabic Language, reviewed, and then validated by two family medicine consultants.

The study questionnaire consisted of caregiver's consent, demographic data of students and their caregivers, estimated number of screen-time hours spent by the student, and associated factors assessment.

\section{Data Collection Technique}

The study questionnaires were distributed directly to students by a female assistant data collector in girls' schools, while a male assistant data collector helped to collect data in boys' schools. Students were asked to take the questionnaires to their home to obtain the consent from their caregivers and to fill the questionnaires, and to bring the filled questionnaires back next day to the assistant data collectors.

\author{
Study Variables \\ Dependent variables: \\ Hours of screen-time. \\ Independent variables: \\ 1. Age \\ 2. Nationality \\ 3. Monthly income \\ 4. Worker mother \\ 5. Last year grade point average (GPA). \\ 6. Hours of physical activity. \\ 7. Sleep hours.
}

\section{Data entry and analysis}

The Statistical Package for Social Sciences (SPSS version 25) was used for data entry and statistical analysis. Pvalues less than 0.05 were considered as statistically significant.

\section{Pilot study / Pretesting:}

A pilot study was performed on 13 students who were not included within the actual study. The Questionnaire had been pretested and the necessary modifications were done.

\section{Ethical considerations:}

The following official approvals were fulfilled by the researcher before start of data collection:

1. Institutional Research Board (IRB).

2. Higher educational authority.

3. Written consents from all participants' caregivers. 
Participant students were assured by the researcher that their responses should not be shared with others. Moreover, anonymity of responses was considered to guarantee honest and more accurate self-reporting.

\section{Relevance and Expectations:}

1. Increase the awareness. Therefore, the risk of screen time will decrease.

2. To adjust the role of media in our society.

3. Establish alternative activities for children and adolescent.

\section{Study difficulties:}

1. Collecting the Data from male schools.

2. Caregiver consent should be taken.

\section{Budget:}

The current study was completely self-funded by the researcher.

Results:-

Table 1:-Personal characteristics of participant students

\begin{tabular}{|l|c|c|}
\hline \multicolumn{1}{|c|}{ Characteristics } & No. & $\%$ \\
\hline Age groups & & 74.3 \\
\hline$\bullet 12-14$ Years & 430 & 25.7 \\
\hline$\bullet 15-16$ years & 85 & 48.6 \\
\hline Gender & 90 & 51.4 \\
\hline$\bullet$ Male & & \\
\hline$\bullet$ Female & 131 & 74.9 \\
\hline Nationality & 44 & 25.1 \\
\hline$\bullet$ Saudi & & \\
\hline$\bullet$ Non-Saudi & 41 & 23.4 \\
\hline Grade & 79 & 45.1 \\
\hline$\bullet 1^{\text {st }}$ & 55 & 31.4 \\
\hline$\bullet 2^{\text {nd }}$ & & \\
\hline$\bullet 3^{\text {rd }}$ & 34 & \\
\hline Grade Point Average (GPA) & 46 & \\
\hline$\bullet>95 \%$ & 38 & 26.3 \\
\hline$\bullet 91-95 \%$ & 35 & 21.7 \\
\hline$\bullet 86-90 \%$ & 22 & 12.6 \\
\hline$\bullet 81-85 \%$ & & \\
\hline$\bullet \leq 8 \%$ & & \\
\hline
\end{tabular}

Table (1) shows that $74.3 \%$ of students aged $12-14$ years, while $25.7 \%$ of students were $15-16$ years old. More than half of students $(51.4 \%)$ were females. Most students were Saudi (74.9\%). About one fourth of students $(23.4 \%)$ were at the first intermediate grade, while 45.1 were at the second grade and $31.4 \%$ were at the third grade. Regarding students' GPA, 19.4\% of students had GPA above 95\%, 26.3\% had 91-95\%, 21.7\% had 86-90\%, 20\% had $81-85 \%$, while $12.6 \%$ had $<80 \%$.

Table 2:-Personal characteristics of students' care takers

\begin{tabular}{|c|c|c|}
\hline \multicolumn{1}{|c|}{ Characteristics } & No. & $\%$ \\
\hline Care taker & & \\
\hline$\bullet$ Parent & 168 & 96.0 \\
\hline$\bullet$ Non-Parent & 7 & 4.0 \\
\hline Age groups (in years) & 30 & \\
\hline$\bullet 30-40$ & 106 & 17.1 \\
\hline$\bullet 41-50$ & 30 & 60.6 \\
\hline$\bullet 51-60$ & & 17.1 \\
\hline
\end{tabular}




\begin{tabular}{|l|c|c|}
\hline$\bullet>60$ & 9 & 5.1 \\
\hline Gender & & 88.6 \\
\hline$\bullet$ Male & 155 & 11.4 \\
\hline$\bullet$ Female & 20 & 1.1 \\
\hline Marital status & 2 & 93.1 \\
\hline$\bullet$ Single & 163 & 2.9 \\
\hline$\bullet$ Married & 5 & 2.9 \\
\hline$\bullet$ Divorced & 5 & \\
\hline$\bullet$ Widowed & & 4.6 \\
\hline Educational level & 8 & 30.9 \\
\hline$\bullet$ Illiterate & 54 & 5.1 \\
\hline$\bullet$ High school & 9 & 51.4 \\
\hline$\bullet$ Vocational/Technical School & 90 & 8.0 \\
\hline$\bullet$ University Degree & 14 & \\
\hline$\bullet$ Postgraduate Degree & & 58.9 \\
\hline Employment status & 103 & 16.0 \\
\hline$\bullet$ Full-Time & 28 & 25.1 \\
\hline$\bullet$ Part-Time & 44 & \\
\hline$\bullet$ Student & & \\
\hline
\end{tabular}

Table (2) shows that $96 \%$ of students' care takers were their parents. About two thirds of care takers (60.6\%) aged 41-50 years, $17.1 \%$ were $30-40$ years old, $17.1 \%$ were $51-60$ years old, while $5.1 \%$ were $>60$ years old. Most care takers were males $(88.6 \%)$. The majority of care takers $(93.1 \%)$ were married, while $1.1 \%$ were single, $2.9 \%$ were divorced and $2.9 \%$ were widowed. More than half of care takers $(51.4 \%)$ were university graduates, while $30.9 \%$ had high school degree, $4.6 \%$ were illiterate, $5.1 \%$ had vocational/technical school education and $8 \%$ had postgraduate educational degrees. Most care takers (58.9\%) were full-time employed, while $16 \%$ were part-time employed and $25.1 \%$ were students.

Table 3:-Characteristics of students' families

\begin{tabular}{|l|c|c|}
\hline \multicolumn{1}{|c|}{ Characteristics } & No. & $\%$ \\
\hline Household income & 38 & 21.7 \\
\hline$\bullet<5000$ SR & 77 & 44.0 \\
\hline$\bullet 5000-9999$ SR & 52 & 29.7 \\
\hline$\bullet 10,000-29999$ SR & 8 & \\
\hline$\bullet>30,000$ SR & & 28.0 \\
\hline No. of children & 49 & 48.6 \\
\hline$\bullet 1-3$ & 85 & 19.4 \\
\hline$\bullet 4-6$ & 34 & 4.0 \\
\hline$\bullet 7-10$ & 7 & 3.4 \\
\hline$\bullet>10$ & & 45.1 \\
\hline Family size & 6 & 42.9 \\
\hline$\bullet<4$ & 79 & 8.6 \\
\hline$\bullet 4-6$ & 75 & \\
\hline$\bullet 7-10$ & 15 & 5.1 \\
\hline$\bullet>10$ & & 8.0 \\
\hline Child living with: & 9 & \\
\hline$\bullet$ Father & 14 & 23.4 \\
\hline$\bullet$ Mother & 152 & 76.6 \\
\hline$\bullet$ Both parents & & \\
\hline Mother's employment & 41 & \\
\hline$\bullet$ Employed & 134 & \\
\hline$\bullet$ Housewife & & \\
\hline
\end{tabular}


Table (3) shows that $21.7 \%$ of students' household income was less than 5000 SR, 44\% had 5000-9999 SR, 29.7\% had 10,000-29999 SR and 4.6 had 30,000 SR or more. Almost half of students' families (48.6\%) had 4-6 children, $28 \%$ had $1-3$ children, $19.4 \%$ had $7-10$ children, while $4 \%$ had more than 10 children. The family size of $45.1 \%$ of students was $4-6$, that of $42.9 \%$ was $7-10$, while that of $3.4 \%$ was <4 and that of $8.6 \%$ was more than 10 . The majority of students were living with their parents $(86.9 \%)$, while $5.1 \%$ were living with their fathers and $8 \%$ were living with their mothers. Most students' mothers were housewives (76.6\%), while 23.4\% were employed.

Table 4:-Characteristics of using electronic devices by students

\begin{tabular}{|c|c|c|}
\hline Characteristics & No. & $\%$ \\
\hline \multicolumn{3}{|l|}{ Using electronic devices } \\
\hline - Yes & 125 & 71.4 \\
\hline - $\mathrm{No}$ & 50 & 28.6 \\
\hline \multicolumn{3}{|l|}{ Average duration of daily use electronic devices } \\
\hline - None & 50 & 28.6 \\
\hline - $\leq 1$ hour & 75 & 42.9 \\
\hline - 2 - 4 hours & 45 & 25.7 \\
\hline - 4- 6 hours & 3 & 1.7 \\
\hline - $>8$ hours & 2 & 1.1 \\
\hline \multicolumn{3}{|l|}{ Used electronic devices } \\
\hline - Smartphone & 122 & 69.7 \\
\hline - Television & 92 & 52.6 \\
\hline - Videogames & 91 & 52.0 \\
\hline - Tablet & 70 & 40.0 \\
\hline - Laptop/Computer & 56 & 32.0 \\
\hline - Cellular phone & 19 & 10.9 \\
\hline
\end{tabular}

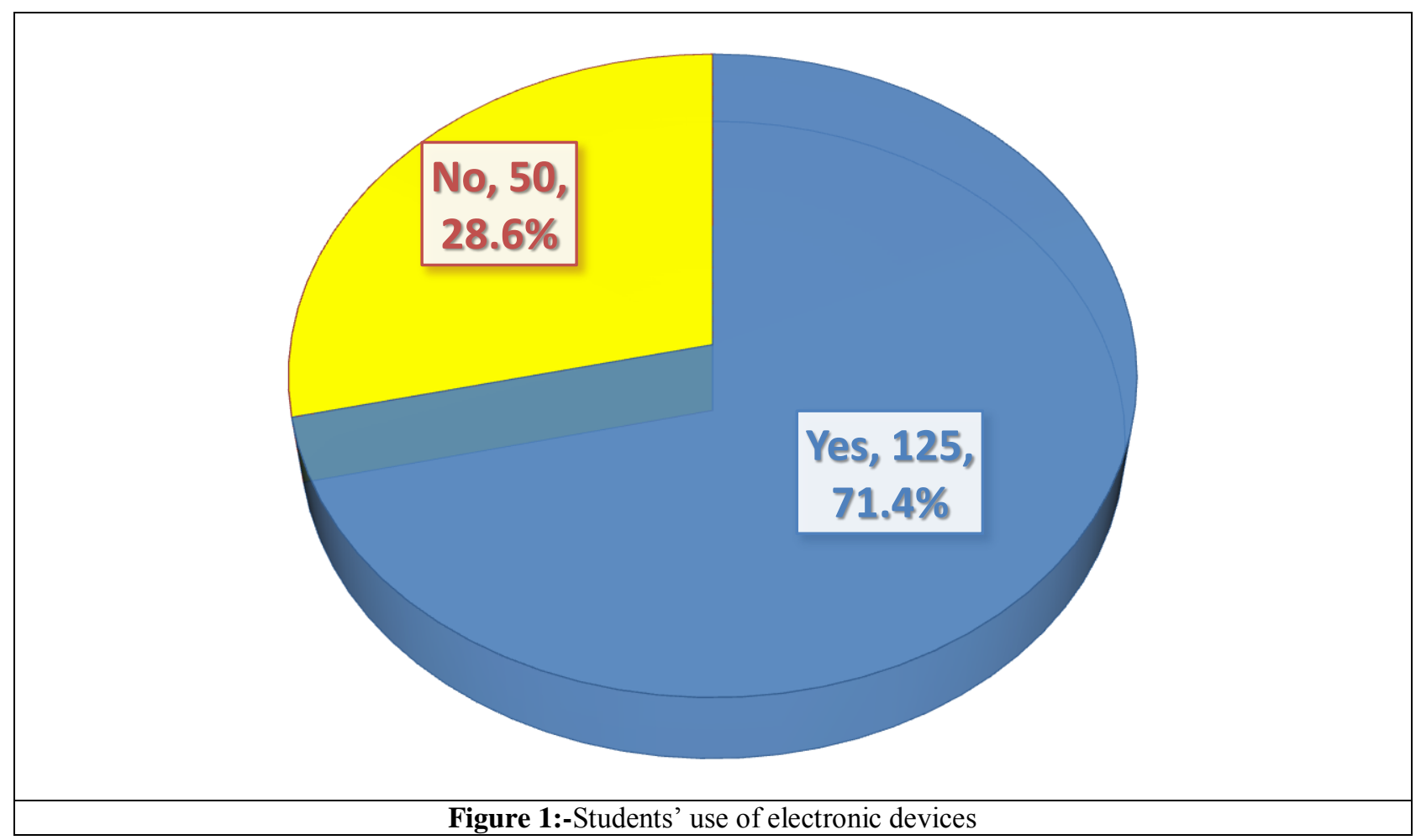




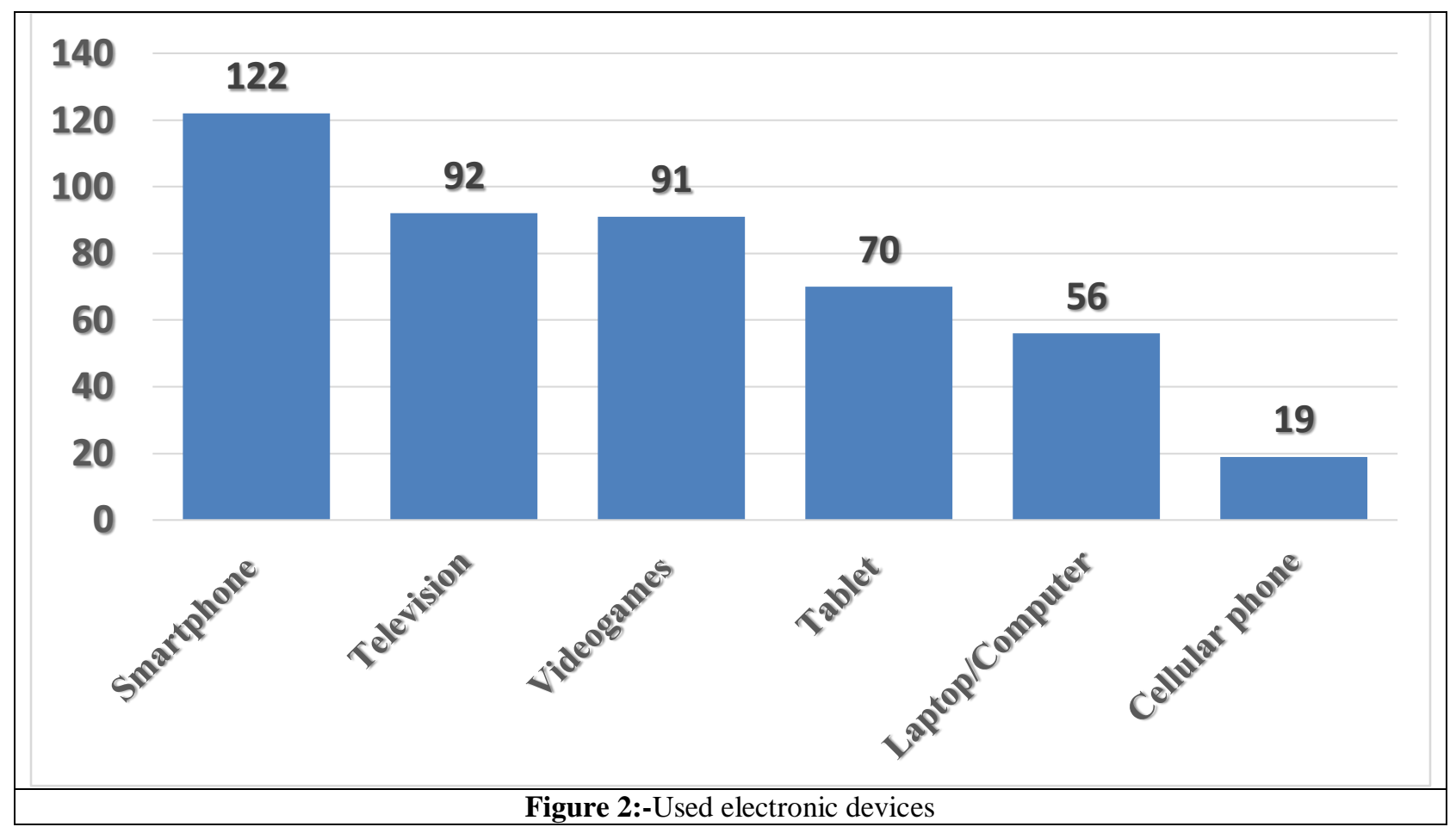

Table (4) shows that $71.4 \%$ of students use electronic devices (Figure 1). The average duration of daily use of electronic devices was $<1$ hour among $42.9 \%$ of students, $2-4$ hours among $25.7 \%$ of students, while it was $4-6$ hours among $1.7 \%$ and $>7$ hours among $1.1 \%$. The smartphones were the most frequently used electronic devices $(69.7 \%)$, followed by the television $(52.6 \%)$, videogames $(52 \%)$, tablets $(40 \%)$, laptops/computers $(32 \%)$ and the cellular phones (10.9\%), as shown in Figure (2).

Table 5:-Frequency of students' different electronic devices activities during weekdays and weekends

\begin{tabular}{|c|c|c|c|c|c|c|c|c|c|c|}
\hline & \multicolumn{2}{|c|}{ Not used } & \multicolumn{2}{|c|}{$<1$ hour } & \multicolumn{2}{|c|}{ 2-4 hours } & \multicolumn{2}{|c|}{ 5-7 hours } & \multicolumn{2}{|c|}{$>8$ hours } \\
\hline Activities & No. & $\%$ & No. & $\%$ & No. & $\%$ & No. & $\%$ & No. & $\%$ \\
\hline \multicolumn{11}{|c|}{ Using electronic devices: } \\
\hline - Weekdays & 43 & 24.6 & 31 & 17.7 & 63 & 36.0 & 28 & 16.0 & 10 & 5.7 \\
\hline - Weekends & 32 & 18.3 & 6 & 3.4 & 49 & 28.0 & 51 & 29.1 & 37 & 21.1 \\
\hline & \multicolumn{10}{|c|}{$\chi^{2}=42.46 \mathrm{p}<0.001$} \\
\hline \multicolumn{11}{|c|}{ Educational devices: } \\
\hline - Weekdays & 39 & 22.3 & 94 & 53.7 & 35 & 20.0 & 4 & 2.3 & 3 & 1.7 \\
\hline - Weekends & 100 & 57.1 & 38 & 21.7 & 28 & 16.0 & 7 & 4.0 & 2 & 1.1 \\
\hline & \multicolumn{10}{|c|}{$\chi^{2}=52.32 \mathrm{p}<0.001$} \\
\hline \multicolumn{11}{|c|}{ Entertainment devices: } \\
\hline - Weekdays & 44 & 25.1 & 37 & 21.1 & 68 & 38.9 & 17 & 9.7 & 9 & 5.2 \\
\hline - Weekends & 49 & 28.0 & 8 & 4.6 & 52 & 29.7 & 47 & 26.9 & 20 & 11.4 \\
\hline & \multicolumn{10}{|c|}{$\chi^{2}=39.32 \mathrm{p}<0.001$} \\
\hline \multicolumn{11}{|l|}{ Texting devices: } \\
\hline - Weekdays & 77 & 44.0 & 65 & 37.1 & 19 & 10.9 & 9 & 5.1 & 5 & 2.8 \\
\hline - Weekends & 84 & 48.0 & 38 & 21.7 & 31 & 17.7 & 12 & 6.9 & 10 & 5.7 \\
\hline & \multicolumn{10}{|c|}{$\chi^{2}=12.36 \mathrm{p}=0.016$} \\
\hline \multicolumn{11}{|l|}{ Phone calls: } \\
\hline - Weekdays & 116 & 66.3 & 47 & 26.9 & 6 & 3.4 & 4 & 2.3 & 2 & 1.1 \\
\hline - Weekends & 126 & 72.0 & 33 & 18.9 & 10 & 5.7 & 4 & 2.3 & 2 & 1.1 \\
\hline & \multicolumn{10}{|c|}{$\chi^{2}=3.86 \mathrm{p}=0.426$} \\
\hline
\end{tabular}




\begin{tabular}{|c|c|c|c|c|c|c|c|c|c|c|}
\hline - Weekdays & 65 & 36.6 & 63 & 36.0 & 37 & 21.1 & 8 & 4.6 & 2 & 1.1 \\
\hline - Weekends & 78 & 44.6 & 42 & 24.0 & 36 & 20.6 & 17 & 9.7 & 2 & 1.1 \\
\hline & \multicolumn{10}{|c|}{$\chi^{2}=8.64 p=0.073$} \\
\hline \multicolumn{11}{|l|}{ Chatting: } \\
\hline - Weekdays & 88 & 50.3 & 49 & 28.0 & 6 & 3.4 & 10 & 5.7 & 0 & 0.0 \\
\hline - Weekends & 88 & 50.3 & 28 & 16.0 & 31 & 17.7 & 24 & 13.7 & 4 & 2.3 \\
\hline & \multicolumn{10}{|c|}{$\chi^{2}=31.05 \mathrm{p}<0.001$} \\
\hline \multicolumn{11}{|c|}{ Using electronic devices while transit } \\
\hline - Weekdays & 126 & 72.0 & 41 & 23.4 & 4 & 2.3 & 4 & 2.3 & 0 & 0.0 \\
\hline - Weekends & 114 & 65.1 & 47 & 26.9 & 8 & 4.6 & 6 & 3.4 & 0 & 0.0 \\
\hline & \multicolumn{10}{|c|}{$\chi^{2}=2.74 p=0.434$} \\
\hline
\end{tabular}

Table (5) shows that use of electronic devices increases significantly at weekends ( $\mathrm{p}<0.001)$. Moreover, educational and entertainment use of electronic devices differed significantly during weekends from that during weekdays $(\mathrm{p}<0.001$ for both). Texting and chatting also differed significantly during weekdays from that during weekends $(\mathrm{p}=0.016$ and $\mathrm{p}<0.001$, respectively). However, making phone calls, watching TV and using electronic devices while transit did not differ significantly during weekdays from that during weekends. Entertainment was the most frequent purpose for using electronic devices by participant students for more than 8 hours.

Table 6:-Students' practices of physical activity and sleep during weekdays and weekends

\begin{tabular}{|c|c|c|c|c|c|c|c|c|c|c|}
\hline & \multicolumn{2}{|c|}{ Not practiced } & \multicolumn{2}{|c|}{$<1$ hour } & \multicolumn{2}{|c|}{ 2-4 hours } & \multicolumn{2}{|c|}{ 5-7 hours } & \multicolumn{2}{|c|}{$>8$ hours } \\
\hline Practices & No. & $\%$ & No. & $\%$ & No. & $\%$ & No. & $\%$ & No. & $\%$ \\
\hline \multicolumn{11}{|l|}{ Physical activity } \\
\hline - Weekdays & 68 & 38.9 & 67 & 38.3 & 31 & 17.7 & 9 & 5.1 & 0 & 0.0 \\
\hline - Weekends & 77 & 44.0 & 40 & 22.9 & 42 & 24.0 & 11 & 6.3 & 16 & 9.1 \\
\hline & \multicolumn{10}{|c|}{$\chi^{2}=24.92 p<0.001$} \\
\hline \multicolumn{11}{|l|}{ Duration of sleep } \\
\hline - Weekdays & 0 & 0.0 & 0 & 0.0 & 0 & 0.0 & 97 & 55.4 & 78 & 44.6 \\
\hline - Weekends & 0 & 0.0 & 0 & 0.0 & 0 & 0.0 & 97 & 55.4 & 78 & 44.6 \\
\hline & \multicolumn{10}{|c|}{$\chi^{2}=0.0 \mathrm{p}=0.100$} \\
\hline
\end{tabular}

Table (6) shows that prolonged ( $>8$ hours) physical activity was significantly more during weekends $(9.1 \%$ vs. $0 \%$, $\mathrm{p}<0.001)$. On the other hand, duration of sleep did not differ during weekdays and weekends.

Table 7:-Use of electronic devices for educational activities during weekdays and weekends

\begin{tabular}{|c|c|c|c|c|c|c|c|c|c|c|}
\hline Electronic & \multicolumn{2}{|c|}{ Not used } & \multicolumn{2}{|c|}{$<1$ hour } & \multicolumn{2}{|c|}{ 2-4 hours } & \multicolumn{2}{|c|}{ 5-7 hours } & \multicolumn{2}{|c|}{$>8$ hours } \\
\hline Devices & No. & $\%$ & No. & $\%$ & No. & $\%$ & No. & $\%$ & No. & $\%$ \\
\hline \multicolumn{11}{|l|}{ Television } \\
\hline - Weekdays & 94 & 53.7 & 49 & 28.0 & 26 & 14.9 & 4 & 2.3 & 2 & 1.1 \\
\hline - Weekends & 100 & 57.1 & 22 & 12.6 & 36 & 20.6 & 12 & 6.9 & 5 & 2.9 \\
\hline & \multicolumn{10}{|c|}{$\chi^{2}=17.35 \mathrm{p}=0.002$} \\
\hline \multicolumn{11}{|l|}{ MP3 } \\
\hline - Weekdays & 145 & 82.9 & 19 & 10.9 & 6 & 3.4 & 5 & 2.9 & 0 & 0.0 \\
\hline - Weekends & 139 & 79.4 & 15 & 8.6 & 14 & 8.0 & 7 & 4.0 & 0 & 0.0 \\
\hline & \multicolumn{10}{|c|}{$\chi^{2}=4.13 \quad p=0.249$} \\
\hline \multicolumn{11}{|l|}{ Smartphones } \\
\hline - Weekdays & 76 & 43.4 & 38 & 21.7 & 44 & 25.1 & 17 & 9.7 & 0 & 0.0 \\
\hline - Weekends & 79 & 45.1 & 17 & 9.7 & 47 & 26.9 & 32 & 18.3 & 0 & 0.0 \\
\hline & \multicolumn{10}{|c|}{$\chi^{2}=12.77 p=0.006$} \\
\hline \multicolumn{11}{|l|}{ Tablets } \\
\hline - Weekdays & 125 & 71.4 & 31 & 17.7 & 11 & 6.3 & 8 & 4.6 & 0 & 0.0 \\
\hline - Weekends & 127 & 72.6 & 18 & 10.3 & 20 & 11.4 & 10 & 5.7 & 0 & 0.0 \\
\hline & \multicolumn{10}{|c|}{$\chi^{2}=6.30 \mathrm{p}=0.099$} \\
\hline Computer & & & & & & & & & & \\
\hline
\end{tabular}




\begin{tabular}{|l|c|c|c|c|c|c|c|c|c|c|}
\hline - Weekdays & 113 & 64.6 & 42 & 24.0 & 16 & 9.1 & 4 & 2.3 & 0 & 0.0 \\
\hline - Weekends & 126 & 72.0 & 21 & 12.0 & 20 & 11.4 & 8 & 4.6 & 0 & 0.0 \\
\hline & \multicolumn{7}{|c|}{$\chi^{2}=9.48 \mathrm{p}=0.024$} \\
\hline Others & & & & & & & & & & \\
\hline - Weekdays & 169 & 96.6 & 2 & 1.1 & 3 & 1.7 & 1 & 0.6 & 0 & 0.0 \\
\hline - Weekends & 171 & 97.7 & 0 & 0.0 & 2 & 1.1 & 2 & 1.1 & 0 & 0.0 \\
\hline
\end{tabular}

Table (7) shows that use of TV, smartphones and computers for educational activities during weekdays was significantly different from that during weekends $(\mathrm{p}=0.002, \mathrm{p}=0.006$ and $\mathrm{p}=0.024$, respectively). However, use of MP3, tablets and other electronic devices for educational activities during weekdays did not differ significantly different from that during weekends

Table 8:-Students' use of electronic devices for entertainment during weekdays and weekends

\begin{tabular}{|c|c|c|c|c|c|c|c|c|c|c|}
\hline Electronic & \multicolumn{2}{|c|}{ Not used } & \multicolumn{2}{|c|}{$<1$ hour } & \multicolumn{2}{|c|}{ 2-4 hours } & \multicolumn{2}{|c|}{ 5-7 hours } & \multicolumn{2}{|c|}{$>8$ hours } \\
\hline Devices & No. & $\%$ & No. & $\%$ & No. & $\%$ & No. & $\%$ & No. & $\%$ \\
\hline \multicolumn{11}{|l|}{ Television } \\
\hline - Weekdays & 89 & 50.9 & 47 & 26.9 & 27 & 15.4 & 12 & 6.9 & 0 & 0.0 \\
\hline - Weekends & 82 & 46.9 & 25 & 14.3 & 44 & 25.1 & 24 & 13.7 & 0 & 0.0 \\
\hline & \multicolumn{10}{|c|}{$\chi^{2}=15.08 \mathrm{p}=0.002$} \\
\hline \multicolumn{11}{|l|}{ MP3 } \\
\hline - Weekdays & 143 & 81.7 & 20 & 11.4 & 7 & 4.0 & 2 & 1.1 & 3 & 1.7 \\
\hline - Weekends & 135 & 77.1 & 14 & 8.0 & 21 & 12.0 & 2 & 1.1 & 3 & 1.7 \\
\hline & \multicolumn{10}{|c|}{$\chi^{2}=8.29 \mathrm{p}=0.083$} \\
\hline \multicolumn{11}{|l|}{ Smartphones } \\
\hline - Weekdays & 85 & 48.6 & 37 & 21.1 & 33 & 18.9 & 12 & 6.9 & 8 & 4.6 \\
\hline - Weekends & 76 & 43.4 & 15 & 8.6 & 47 & 26.9 & 20 & 11.4 & 17 & 9.7 \\
\hline & \multicolumn{10}{|c|}{$\chi^{2}=17.50 \mathrm{p}=0.002$} \\
\hline \multicolumn{11}{|l|}{ Tablets } \\
\hline - Weekdays & 137 & 78.3 & 21 & 12.0 & 10 & 5.7 & 4 & 2.3 & 3 & 1.7 \\
\hline - Weekends & 123 & 70.3 & 18 & 10.3 & 23 & 13.1 & 6 & 3.4 & 5 & 2.9 \\
\hline & \multicolumn{10}{|c|}{$\chi^{2}=7.01 \mathrm{p}=0.137$} \\
\hline \multicolumn{11}{|l|}{ Computer } \\
\hline - Weekdays & 127 & 72.6 & 34 & 19.4 & 9 & 5.1 & 2 & 1.1 & 3 & 1.7 \\
\hline - Weekends & 125 & 71.4 & 23 & 13.1 & 19 & 10.9 & 1 & 0.6 & 7 & 4.0 \\
\hline & \multicolumn{10}{|c|}{$\chi^{2}=7.64 \mathrm{p}=0.107$} \\
\hline \multicolumn{11}{|l|}{ Others } \\
\hline - Weekdays & 168 & 96.0 & 1 & .6 & 4 & 2.3 & 1 & .6 & 1 & .6 \\
\hline - Weekends & 169 & 96.6 & 1 & .6 & 3 & 1.7 & 1 & .6 & 1 & .6 \\
\hline & \multicolumn{10}{|c|}{$\chi^{2}=0.15 \mathrm{p}=0.997$} \\
\hline
\end{tabular}

Table (8) shows that use of TV and smartphones for entertainment activities during weekdays was significantly different from that during weekends ( $\mathrm{p}=0.002$, for both). However, use of other electronic devices for entertainment activities during weekdays did not differ significantly different from that during weekends

Table 9:-Frequency of having meals with families during weekdays and weekends

\begin{tabular}{|c|c|c|}
\hline No. of meals with families & No. & $\%$ \\
\hline \multicolumn{3}{|l|}{ During weekdays } \\
\hline - None & 2 & 1.1 \\
\hline - One & 6 & 3.4 \\
\hline - Two & 59 & 33.7 \\
\hline - Three & 103 & 58.9 \\
\hline \multicolumn{3}{|l|}{ During weekends } \\
\hline - None & 7 & 4.0 \\
\hline - One & 6 & 3.4 \\
\hline
\end{tabular}




\begin{tabular}{|c|c|c|}
\hline - Two & 59 & 33.7 \\
\hline - Three & 103 & 58.9 \\
\hline
\end{tabular}

Table (9) shows that more than half of students have 3 meals with their families during weekdays and weekends (58.9\% for both).

Table 10:-Frequency of using electronic devices while having meals during weekdays and weekends

\begin{tabular}{|l|c|c|}
\hline \multicolumn{1}{|c|}{ Use of electronic devices while having meals } & No. \\
\hline During weekdays & & \\
\hline$\bullet$ Never & 106 & 60.6 \\
\hline$\bullet$ Sometimes & 41 & 23.4 \\
\hline$\bullet$ Often & 18 & 10.3 \\
\hline$\bullet$ Always & 10 & 5.7 \\
\hline During weekends & & \\
\hline$\bullet$ Never & 92 & 52.6 \\
\hline$\bullet$ Sometimes & 41 & 23.4 \\
\hline$\bullet$ Often & 18 & 10.3 \\
\hline$\bullet$ Always & 24 & 13.7 \\
\hline
\end{tabular}

$\chi^{2}=6.75 \quad \mathrm{P}=0.082$

Table (10) shows that using electronic devices while having meals during weekdays did not differ significantly from that during weekends.

Table 11:-Watching TV watching during meals during weekdays and weekends

\begin{tabular}{|l|c|c|}
\hline \multicolumn{1}{|c|}{ TV watching during meals } & No. & $\%$ \\
\hline During weekdays & & \\
\hline$\bullet$ Never & 74 & 42.3 \\
\hline$\bullet$ Sometimes & 65 & 37.1 \\
\hline$\bullet$ Often & 19 & 10.9 \\
\hline$\bullet$ Always & 17 & 9.7 \\
\hline During weekends & & \\
\hline$\bullet$ Never & 72 & 41.1 \\
\hline$\bullet$ Sometimes & 64 & 36.6 \\
\hline$\bullet$ Often & 16 & 9.1 \\
\hline$\bullet$ Always & 23 & 13.1 \\
\hline
\end{tabular}

$$
\chi^{2}=1.19 \quad \mathrm{P}=0.755
$$

Table (11) shows that more than half of students watch TV during meals, both during weekdays and weekends. However, watching TV during meals during weekdays did not differ significantly from that during weekends.

Table 12:-Students' use of electronic devices according to their practice of physical activity, duration of sleep and number of meals of students with their families

\begin{tabular}{|c|c|c|c|c|c|}
\hline \multirow{3}{*}{ Variables } & \multicolumn{4}{|c|}{ Use of electronic devices } & \multirow{3}{*}{$\frac{\mathrm{P}}{\text { Value }}$} \\
\hline & \multicolumn{2}{|c|}{ Yes } & \multicolumn{2}{|c|}{ No } & \\
\hline & No. & $\%$ & No. & $\%$ & \\
\hline \multicolumn{6}{|l|}{ Physical activity during weekdays } \\
\hline - No & 47 & 69.1 & 21 & 30.9 & $\chi^{2}=0.291$ \\
\hline - Yes & 78 & 72.9 & 29 & 27.1 & $\mathrm{P}=0.590$ \\
\hline \multicolumn{6}{|l|}{ Physical activity during weekends } \\
\hline - No & 53 & 68.8 & 24 & 31.2 & $\chi^{2}=0.455$ \\
\hline - Yes & 72 & 73.5 & 26 & 26.5 & $\mathrm{P}=0.500$ \\
\hline \multicolumn{6}{|l|}{ Duration of sleep during weekdays } \\
\hline - $<8$ hours & 69 & 71.1 & 28 & 28.9 & $\chi^{2}=0.009$ \\
\hline - $>8$ hours & 56 & 71.8 & 22 & 28.2 & $\mathrm{P}=0.923$ \\
\hline
\end{tabular}




\begin{tabular}{|l|r|r|r|r|r|}
\hline Duration of sleep during weekends & & & & & \\
\hline$\bullet<8$ hours & 66 & 68.0 & 31 & 32.0 & $\chi^{2}=1.224$ \\
\hline$\bullet>8$ hours & 59 & 75.6 & 19 & 24.4 & $\mathrm{P}=0.269$ \\
\hline No. of meals with families during weekdays & & & & & \\
\hline$\bullet$ None & 1 & 50.0 & 1 & 50.0 & \\
\hline$\bullet$ One & 18 & 78.3 & 5 & 21.7 & $\chi^{2}=1.456$ \\
\hline$\bullet$ Two & 52 & 73.2 & 19 & 26.8 & $\mathrm{P}=0.692$ \\
\hline$\bullet$ Three & 54 & 68.4 & 25 & 31.6 & \\
\hline No. of meals with families during weekends & & & & & \\
\hline$\bullet$ None & 5 & 71.4 & 2 & 28.6 & \\
\hline$\bullet$ One & 5 & 83.3 & 1 & 16.7 & $\chi^{2}=1.018$ \\
\hline$\bullet$ Two & 44 & 74.6 & 15 & 25.4 & $\mathrm{P}=0.797$ \\
\hline$\bullet$ Three & 71 & 68.9 & 32 & 31.1 & \\
\hline
\end{tabular}

Table (12) shows that use of electronic devices did not differ significantly according to students' practice of physical activity, duration of sleep or number of meals shared with their families.

Table 13:-Students' use of electronic devices according to their personal characteristics

\begin{tabular}{|l|c|c|c|c|c|}
\hline \multicolumn{1}{|c|}{ Personal Characteristics } & \multicolumn{3}{|c|}{ Use of electronic devices } & \\
\hline \multicolumn{1}{|c|}{ No. } & $\%$ & No. & $\%$ & Value \\
\hline Age groups & & & & & \\
\hline$\bullet 12-14$ Years & 95 & 73.1 & 35 & 26.9 & $\chi^{2}=0.673$ \\
\hline$\bullet 15-16$ years & 30 & 66.7 & 15 & 33.3 & $\mathrm{P}=0.412$ \\
\hline Gender & & & & & \\
\hline$\bullet$ Male & 67 & 78.8 & 18 & 21.2 & $\chi^{2}=4.429$ \\
\hline$\bullet$ Female & 58 & 64.4 & 32 & 35.6 & $\mathrm{P}=0.035$ \\
\hline Nationality & & & & & \\
\hline$\bullet$ Saudi & 92 & 70.2 & 39 & 29.8 & $\chi^{2}=0.367$ \\
\hline$\bullet$ Non-Saudi & 33 & 75.0 & 11 & 25.0 & $\mathrm{P}=0.544$ \\
\hline Grade & & & & & \\
\hline$\bullet 1^{\text {st }}$ & 29 & 70.7 & 12 & 29.3 & \\
\hline$\bullet 2^{\text {nd }}$ & 59 & 74.7 & 20 & 25.3 & $\chi^{2}=0.885$ \\
\hline$\bullet 3^{\text {rd }}$ & 37 & 67.3 & 18 & 32.7 & $\mathrm{P}=0.642$ \\
\hline Grade Point Average (GPA) & & & & & \\
\hline$\bullet>95 \%$ & 29 & 85.3 & 5 & 14.7 & \\
\hline$\bullet 91-95 \%$ & 33 & 71.7 & 13 & 28.3 & \\
\hline$\bullet 86-90 \%$ & 26 & 68.4 & 12 & 31.6 & $\chi^{2}=11.206$ \\
\hline$\bullet 81-85 \%$ & 27 & 77.1 & 8 & 22.9 & $\mathrm{P}=0.024$ \\
\hline$\bullet \leq 80 \%$ & 10 & 45.5 & 12 & 54.5 & \\
\hline
\end{tabular}

Table (13) shows that use of electronic devices was significantly higher among male students than female students ( $78.8 \%$ and $64.4 \%$, respectively, $\mathrm{p}=0.035$ ). Moreover, students whose GPA was $>95 \%$ had significantly highest use of electronic devices $(85.3 \%, \mathrm{p}=0.024)$. However, use of electronic devices did not differ significantly according to students' age groups, nationality or grade.

Table 14:-Students' use of electronic devices according to personal characteristics of their care takers

\begin{tabular}{|l|c|c|c|c|c|}
\hline \multicolumn{1}{|c|}{ Personal Characteristics } & \multicolumn{3}{|c|}{ Use of electronic devices } & No & P \\
\hline & No. & $\%$ & No. & $\%$ & Value \\
\hline Care taker & & & & & \\
\hline - Parent & 120 & 71.4 & 48 & 28.6 & $\chi^{2}=0.000$ \\
\hline
\end{tabular}




\begin{tabular}{|l|c|c|c|c|c|}
\hline$\bullet$ Non-Parent & 5 & 71.4 & 2 & 28.6 & $\mathrm{P}=1.000$ \\
\hline Age groups (in years) & & & & & \\
\hline$\bullet 30-40$ & 20 & 66.7 & 10 & 33.3 & \\
\hline$\bullet 41-50$ & 80 & 75.5 & 26 & 24.5 & $\chi^{2}=2.246$ \\
\hline$\bullet 51-60$ & 19 & 63.3 & 11 & 36.7 & $\mathrm{P}=0.523$ \\
\hline$\bullet>60$ & 6 & 66.7 & 3 & 33.3 & \\
\hline Gender & & & & & \\
\hline$\bullet$ Male & 112 & 72.3 & 43 & 27.7 & $\chi^{2}=0.457$ \\
\hline$\bullet$ Female & 13 & 65.0 & 7 & 35.0 & $\mathrm{P}=0.499$ \\
\hline Marital status & & & & & \\
\hline$\bullet$ Single & 2 & 100.0 & 0 & 0.0 & \\
\hline$\bullet$ Married & 117 & 71.8 & 46 & 28.2 & $\chi^{2}=1.450$ \\
\hline$\bullet$ Divorced & 3 & 60.0 & 2 & 40.0 & $\mathrm{P}=0.694$ \\
\hline$\bullet$ Widowed & 3 & 60.0 & 2 & 40.0 & \\
\hline Educational level & & & & & \\
\hline$\bullet$ Illiterate & 5 & 62.5 & 3 & 37.5 & \\
\hline$\bullet$ High school & 35 & 64.8 & 19 & 35.2 & \\
\hline$\bullet$ Vocational/Technical School & 5 & 55.6 & 4 & 44.4 & $\chi^{2}=4.141$ \\
\hline$\bullet$ University Degree & 69 & 76.7 & 21 & 23.3 & $\mathrm{P}=0.387$ \\
\hline$\bullet$ Postgraduate Degree & 11 & 78.6 & 3 & 21.4 & \\
\hline Employment status & & & & & \\
\hline$\bullet$ Full-Time & 74 & 71.8 & 29 & 28.2 & \\
\hline$\bullet$ Part-Time & 21 & 75.0 & 7 & 25.0 & $\chi^{2}=0.411$ \\
\hline$\bullet$ Student & 30 & 68.2 & 14 & 31.8 & $\mathrm{P}=0.814$ \\
\hline
\end{tabular}

Table (14) shows that students' use of electronic devices did not differ significantly according to their care takers' personal characteristics.

Table 15:-Students' use of electronic devices according to characteristics of their families

\begin{tabular}{|c|c|c|c|c|c|}
\hline \multirow{3}{*}{ Family Characteristics } & \multicolumn{4}{|c|}{ Use of electronic devices } & \multirow{3}{*}{$\frac{\mathrm{P}}{\text { Value }}$} \\
\hline & \multicolumn{2}{|c|}{ Yes } & \multicolumn{2}{|c|}{ No } & \\
\hline & No. & $\%$ & No. & $\%$ & \\
\hline \multicolumn{6}{|l|}{ Household income } \\
\hline - $<5000 \mathrm{SR}$ & 25 & 65.8 & 13 & 34.2 & \\
\hline - $5000-9999 \mathrm{SR}$ & 53 & 68.8 & 24 & 31.2 & $\chi^{2}=2.628$ \\
\hline - $10,000-29999 \mathrm{SR}$ & 40 & 76.9 & 12 & 23.1 & $\mathrm{P}=0.453$ \\
\hline - >30,000 SR & 7 & 87.5 & 1 & 12.5 & \\
\hline \multicolumn{6}{|l|}{ No. of children } \\
\hline - $1-3$ & 37 & 75.5 & 12 & 24.5 & \\
\hline - 4-6 & 63 & 74.1 & 22 & 25.9 & $\chi^{2}=2.957$ \\
\hline - $7-10$ & 21 & 61.8 & 13 & 38.2 & $\mathrm{P}=0.398$ \\
\hline - $>10$ & 4 & 57.1 & 3 & 42.9 & \\
\hline \multicolumn{6}{|l|}{ Family size } \\
\hline - $<4$ & 4 & 66.7 & 2 & 33.3 & \\
\hline - 4-6 & 57 & 72.2 & 22 & 27.8 & $\chi^{2}=0.135$ \\
\hline - $7-10$ & 53 & 70.7 & 22 & 29.3 & $\mathrm{P}=0.987$ \\
\hline - $>10$ & 11 & 73.3 & 4 & 26.7 & \\
\hline \multicolumn{6}{|l|}{ Child living with: } \\
\hline - Father & 5 & 55.6 & 4 & 44.4 & \\
\hline - Mother & 10 & 71.4 & 4 & 28.6 & $\chi^{2}=1.177$ \\
\hline - Both parents & 110 & 72.4 & 42 & 27.6 & $\mathrm{P}=0.555$ \\
\hline Mother's employment & & & & & \\
\hline
\end{tabular}




\begin{tabular}{|l|c|c|c|c|c|}
\hline - Employed & 28 & 68.3 & 13 & 31.7 & $\chi^{2}=0.258$ \\
\hline - Housewife & 97 & 72.4 & 37 & 27.6 & $\mathrm{P}=0.611$ \\
\hline
\end{tabular}

Table (15) shows that students' use of electronic devices did not differ significantly according to their family characteristics.

\section{Discussion:-}

There has been a negative association between health status of adolescents and their spent time watching TV (14). Therefore, preventive health campaigns among early adolescents should focus on reducing any unhealthy behavior, (e.g., prolonged television viewing, and videogame playing) (15).

Since technology-based inactive behaviors change quickly, the association between new types of sedentary behaviors among adolescents needs to be assessed (16). Therefore, the present study aimed to assess the screen-time and to determine the factors associated with it among intermediate school students in Makkah Al-Mokarramah City, Saudi Arabia.

Findings of the present study revealed that almost three-fourths of intermediate school students in Makkah AlMokarramah City use electronic devices, mainly smartphones (69.7\%), TV (52.6\%), videogames (52\%), tablets $(40 \%)$ and laptops or computers (32\%). More than one fourth of students use electronic devices for two hours or more daily.

Stockman (17) stated that excessive TV watching during adolescence can be associated with long-lasting adverse consequences and poor educational achievement.

Similar findings were reported by Patriarca et al. (15) among adolescents aged 11-16 years in the Campania region, South of Italy, who found that $52.5 \%$ of them always watched television, mostly more than two hours daily and the frequency of watching for at least two hours daily $(74.9 \%)$, while two-thirds of the students played videogames for 1.6 hours daily and the computer was used by $85 \%$ of the sample for 1.6 hours daily.

In China, Jiang et al. (18) found that TV watching and playing on the computer were the most prevalent screen time behaviors among adolescents. Playing with mobile phones was less prevalent.

In USA, average time spent daily by adolescents in viewing TV was 3.1 hours, while those of videogames playing and computer using were 1.49 and 1.19 hours, respectively (19).

Moreover, in New Zealand, the average time spent daily by adolescents in viewing TV was 3.1 hours (20).

Results of this study showed that use of electronic devices by students increases during their weekends and entertainment was the most frequent purpose for using electronic devices for more than 8 hours. Moreover, students' use of electronic devices did not differ significantly according to their care takers' personal characteristics (e.g., age group, nationality, or grade), or family characteristics (e.g., household monthly income, family size, or mother's employment). However, use of electronic devices was significantly higher among male than female students. In addition, students with high grade point average (GPA) significantly used more electronic devices, but use of electronic devices did not differ according to students' practice of physical activity, duration of sleep, or number of meals shared with their families.

LeBlanc et al. (21) noted that boys reported significantly more video game/computer usage than girls. Bucksch et al. (22) found that boys have more screen time than girls and that there is more ST on weekends than on weekdays. Similarly, Ye et al. (23) reported that male sex, junior grade, weekends, and presence of parents were significantly associated with prolonged screen time. Hoyos Cillero and Jago (24) attributed the sex difference regarding screen time to the observation that boys usually spend more time on computers and tablets than girls. Moreover, Babey et al. (25) reported that boys usually spend more time watching television than girls.

The lack of significant differences in the present study among students' screen time according to their age may be explained by the relatively narrow age range (12-16 years), while the significantly more screen time during weekends may be explained by the time spent at school during the weekdays, which is totally spared for free 
activities, including use of electronic devices, during weekends. Jago et al. (26) suggested reducing TV-watching time through school-based educational components assisted by parental involvement with students' homework.

Jiang et al. (18) added that health education should include adolescents' parents to raise their awareness regarding health risks associated with high screen time and how they can positively influence their children by enforcing family rules to limit using electronic devices.

The significantly more screen time among participant students in the present study with higher grade point average (GPA) may reflect the educational benefits of electronic devices. However, Jiang et al. (18) emphasized the potential harms of excessive use of computers or tablet devices, even when it is for educational purposes.

Patriarca et al. (15) noted that family structure has an important influence on students' sedentary behavior. Bigger family size is usually associated with more TV watching. Babey et al. (25) added that higher household income is associated with more TV watching, but with less computer use.

Results of the present study can be useful for developing and planning effective future preventive strategies against to minimize screen time among adolescents.

\section{Study Limitations}

The cross-sectional study design of the present study cannot provide definitive establishment of a causal relationship between predictors and outcomes. Moreover, the methodology used for quantifying using electronic devices was self-report by the respondent adolescents.

Despite the fact that anonymity of students' responses was considered, it is likely that the possibility that students may either over-report or underreport information about screen time. However, it has been proved that a selfadministered questionnaire is valid for group comparisons regarding watching TV, playing videogames, or using computer (27).

\section{Conclusions:-}

Based on findings of the present study, it can be concluded that more than one-fourth intermediate school students in Makkah Al-Mokarramah, Saudi Arabia have prolonged daily screen time (above two hours). The most frequently used electronic devices are mainly smartphones, TV, videogames, tablets, and laptops or computers. Use of electronic devices increases during their weekends and entertainment is the most frequent purpose for using electronic devices for more than 8 hours. Screen time is significantly higher among boys and those with high grade point average (GPA). However, screen time does not differ significantly according to students' family characteristics, practice of physical activity, duration of sleep, or number of meals shared with their families.

\section{Recommendations}

Based on findings of this study, the following is recommended:

1. Immediate and comprehensive actions are needed in order to diminish time spent at watching television, playing videogames, and using computers by adolescents.

2. TV-watching time can be minimized through school-based educational components assisted by parental involvement with students' homework.

3. Health education of adolescents and their parents to raise their awareness regarding health risks associated with high screen time and how they can positively influence their children by enforcing family rules to limit using electronic devices.

4. Further prospective studies are needed to verify the relationship between prolonged screen time and students' personal and family characteristics.

\section{References:-}

1. Screen time / definition of screen time by Medical dictionary [Internet]. [cited 2017 Jan 1]. Available from: http://medical-dictionary. thefreedictionary.com/screen+time

2. Strasburger VC, Jordan AB, Donnerstein E. Health effects of media on children and adolescents. Pediatrics [Internet]. 2010 Apr [cited 2016 Dec 21];125(4):756-67. Available from: http://www.ncbi.nlm.nih.gov/ pubmed/20194281 
3. American Academy of Pediatrics Announces New Recommendations for Children's Media Use [Internet]. [cited 2016 Dec 24]. Available from: https://www.aap.org/en-us/about-the-aap/aap-press-room/Pages/ American-Academy-of-Pediatrics-Announces-New-Recommendations-for-Childrens-Media-Use.aspx

4. Gingold JA, Simon AE, Schoendorf KC. Excess Screen Time in US Children: Association With Family Rules and Alternative Activities. Clin Pediatr (Phila) [Internet]. 2014 Jan 1 [cited 2017 Jan 1];53(1):41-50. Available from: http://www.ncbi.nlm.nih.gov/pubmed/23922251

5. Sigman A. Time for a view on screen time. Arch Dis Child [Internet]. 2012 Nov [cited 2017 Jan 1];97(11):935-42. Available from: http://www.ncbi.nlm.nih.gov/pubmed/23044213

6. Steffen LM, Dai S, Fulton JE, Labarthe DR. Overweight in children and adolescents associated with TV viewing and parental weight: Project HeartBeat! Am J Prev Med [Internet]. 2009 Jul [cited 2017 Jan 1];37(1 Suppl):S50-5. Available from: http://www.ncbi.nlm.nih.gov/pubmed/ 19524156.

7. Primack BA, Swanier B, Georgiopoulos AM, Land SR, Fine MJ. Association between media use in adolescence and depression in young adulthood: a longitudinal study. Arch Gen Psychiatry [Internet]. 2009 Feb [cited 2017 Jan 1];66(2):181-8. Available from: http://www.ncbi.nlm.nih.gov/pubmed/19188540

8. Alosaimi FD, Alyahya H, Alshahwan H, Al Mahyijari N, Shaik SA. Smartphone addiction among university students in Riyadh, Saudi Arabia. Saudi Med J [Internet]. 2016 Jun [cited 2017 Jan 1];37(6):675-83. Available from: http://www.ncbi.nlm.nih.gov/pubmed/27279515

9. Al-Ghamdi S. The association between watching television and obesity in children of school-age in Saudi Arabia. J Fam Community Med [Internet]. 2013 May [cited 2017 Jan 1];20(2):83. Available from: http://www.ncbi.nlm.nih.gov/pubmed/23983559

10. محمد عمر محمد ابوالرب CV [Internet]. [cited 2017 Jan 2]. Available from: http://www.kau.edu.sa/CVEn.aspx?Site_ID=0060948\&Lng=AR

11. Al-Hazzaa HM, Abahussain NA, Al-Sobayel HI, Qahwaji DM, Musaiger AO. Physical activity, sedentary behaviors and dietary habits among Saudi adolescents relative to age, gender and region. Int J Behav Nutr Phys Act [Internet]. 2011 Dec 21 [cited 2017 Jan 2];8:140. Available from: http://www.ncbi.nlm.nih.gov/pubmed/22188825

12. Raosoft Sample Size Calculator. http://www.raosoft.com/ samplesize.html

13. https://www.random.org/

14. Davison KK, Marshall SJ, Birch LL. Cross-sectional and longitudinal associations between TV viewing and girls' body mass index, overweight status, and percentage of body fat. J Pediatr 2006; 149:32-7

15. Patriarca A, Di Giuseppe G, Albano L, Marinelli P, Angelillo IF. Use of television, videogames, and computer among children and adolescents in Italy. BMC Public Health 2009, 9:139.

16. Tsujiguchi H, Hori D, Kambayashi Y, Hamagishi T, Asakura H, Mitoma J, et al. Relationship between screen time and nutrient intake in Japanese children and adolescents: a cross-sectional observational study. Environmental Health and Preventive Medicine 2018; 23:34.

17. Stockman JA. Association of Television Viewing During Childhood with Poor Educational Achievement. Yearbook of Pediatrics, 2007; 78-79.

18. Jiang X, Hardy LL, Ding D, Baur LA, Shi H. Recreational Screen-Time Among Chinese Adolescents: A CrossSectional Study. J Epidemiol 2014; 24(5):397-403.

19. Jordan AB, Hersey JC, McDivitt JA, Heitzler CD: Reducing children's television-viewing time: a qualitative study of parents and their children. Pediatrics 2006, 118:e1303-10.

20. Hancox RJ, Milne BJ, Poulton R. Association of television viewing during childhood with poor educational achievement. Arch Pediatr Adolesc Med 2005; 159:614-618.

21. LeBlanc AG, Broyles ST, Chaput JP, Leduc G, Boyer C, Borghese MM, Tremblay MS. Correlates of objectively measured sedentary time and self-reported screen time in Canadian children. Journal of Behavioral Nutrition and Physical Activity 2015; 12:38.

22. Bucksch J, Sigmundova D, Hamrik Z, Troped PJ, Melkevik O, Ahluwalia N, et al. International Trends in Adolescent Screen-Time Behaviors From 2002 to 2010. J Adolesc Health 2016;58(4):417-25.

23. Ye S, Chen L, Wang Q, Li Q. Correlates of screen time among 8-19-year-old students in China. BMC Public Health 2018; 18:467.

24. Hoyos Cillero I, Jago R. Sociodemographic and home environment predictors of screen viewing among Spanish school children. J Public Health (Oxf) 2011; 33: 392-402.

25. Babey SH, Hastert TA, Wolstein J. Adolescents sedentary behaviors: correlates differ for television viewing and computer use. J Adolesc Health. 2013;52(1):70-6

26. Jago R, Stamatakis E, Gama A, Carvalhal IM, Nogueira H, Rosado V, Padez C. Parent and Child ScreenViewing Time and Home Media Environment. Am J Prev Med 2012;43(2):150-158.

27. Kautiainen S, Koivusilta L, Lintonen T, Virtanen SM, Rimpelä A. Use of information and communication technology and prevalence of overweight and obesity among adolescents. Int J Obes (Lond) 2005; 29:925-33. 